\title{
Occurrence of grapevine yellow speckle viroid 1 in autochthonous grapevines in Slovenia
}

\author{
Nataša Štajner ${ }^{1}$ - Aljoša Beber ${ }^{1} \cdot$ Denis Rusjan $^{1} \cdot$ Jernej Jakše ${ }^{1}$ \\ Received: 24 July 2018 / Accepted: 8 September 2018 / Published online: 2 October 2018 \\ (C) Società Italiana di Patologia Vegetale (S.I.Pa.V.) 2018
}

Many autochthonous grapevine cultivars are becoming popular for cultivation and use in breeding programs. Although they are adapted to local conditions and can offer distinctive, marketable wines, their phytosanitary conditions are insufficiently monitored. To obtain an insight into the health status of old autochthonous Slovenian cultivars, we made a reconstruction of the viral population of grapevines cvs 'Cipro', 'Malvazija', 'Pokalca', 'Volovnik', 'Rebula' and 'Poljšakica' from a collection vineyard of the Biotechnical Faculty, University of Ljubljana. A total of 70,902,637 cleaned sequence reads were generated by small RNA sequencing with the Ion Torrent platform. A sequence of grapevine yellow speckle viroid 1 (GYSVd-1, genus Apscaviroid) was detected by executing the VirusDetect pipeline (Zheng et al. 2017) with an alignment of $0.13 \%$ of total reads reaching high average coverage of 5365 $X$. The presence of GYSVd-1 was confirmed in nine plants of the aforementioned cultivars (GenBank accession numbers MG010373-MG010381), with the exception of cv. 'Malvazija', via RT-PCR using the One step RT-PCR-Qiagen kit and primer 342P (5'-CAATCCCCGGAACCCCCGCT-3') and modified primer $341 \mathrm{M}$ (Sahana et al. 2013) designated as 341 M-m (5'-CACTCGCGGGGCGCGTTGGT-3'). Multiple sequence alignment of full-length sequences using ClustalW (ver. 2.1) revealed eighteen polymorphic nucleotide sites, and twelve $(66.7 \%)$ appertained to the Rebula $2 \mathrm{TR}$ isolate
(MG010376). BLASTn sequence alignment against the nonredundant nucleotide GenBank collection revealed that sequence MG010377 is $100 \%$ identical to KY039380, and MG010378 to X87910, both of European origin. The sequences MG010373-MG010375 shared 99.7\% identity with X87919. The most diverse isolate, Rebula 2TR (MG010376), shared $97.8 \%$ identity with an isolate from Japan (AB028466). In addition, all the GYSVd-1 positive grapevines were shown to be simultaneously infected by grapevine pinot gris virus. To our knowledge, this is the first report of the sequence identification of GYSVd-1 in grapevines in Slovenia.

Acknowledgements The authors acknowledge the financial support of the Slovenian Research Agency (ARRS program no. P4-0077).

\section{References}

Sahana AB, Adkar-Purushothama CR, Chennappa G, Zhang ZX, Sreenivasa MY, Sano T (2013) First report of grapevine yellow speckle viroid-1 and hop stunt viroid infecting grapevines (Vitis vinifera) in India. Plant Dis 97(11):1517-1517

Zheng Y, Gao S, Padmanabhan C, Li R, Galvez M, Gutierrez D, Fuentes S, Ling KS, Kreuze J, Fei Z (2017) VirusDetect: an automated pipeline for efficient virus discovery using deep sequencing of small RNAs. Virology 500:130-138
Nataša Štajner

natasa.stajner@bf.uni-lj.si

1 Biotechnical Faculty, Agronomy Department, University of Ljubljana, Jamnikarjeva 101, SI-1000 Ljubljana, Slovenia 